\title{
Community based interventions for the prevention and control of tuberculosis
}

\author{
Ahmed Arshad', Rehana A Salam', Zohra S Lassi', Jai K Das' ${ }^{1}$ Imama Naqvi ${ }^{1}$ and Zulfiqar A Bhutta ${ }^{2,3^{*}}$
}

\begin{abstract}
In 2012, an estimated 8.6 million people developed tuberculosis (TB) and 1.3 million died from the disease. With its recent resurgence with the human immunodeficiency virus (HIV); TB prevention and management has become further challenging. We systematically evaluated the effectiveness of community based interventions (CBI) for the prevention and treatment of TB and a total of 41 studies were identified for inclusion. Findings suggest that CBI for TB prevention and case detection showed significant increase in TB detection rates (RR: 3.1, 95\% Cl: 2.92, 3.28) with non-significant impact on TB incidence. CBI for treating patients with active TB showed an overall improvement in treatment success rates (RR: 1.09, 95\% Cl: $1.07,1.11)$ and evidence from a single study suggests significant reduction in relapse rate (RR: $0.26,95 \% \mathrm{Cl}: 0.18,0.39$ ). The results were consistent for various study design and delivery mechanism. Qualitative synthesis suggests that community based TB treatment delivery through community health workers (CHW) not only improved access and service utilization but also contributed to capacity building and improving the routine TB recording and reporting systems. CBI coupled with the DOTS strategy seem to be an effective approach, however there is a need to evaluate various community-based integrated delivery models for relative effectiveness.
\end{abstract}

Keywords: Community-based interventions, Tuberculosis, DOTS, integrated delivery, CHWs

\section{Multilingual abstracts}

Please see Additional file 1 for translations of the abstract into the six official working languages of the United Nations.

\section{Introduction}

Tuberculosis (TB) remains a major global health problem. In 2012, an estimated 8.6 million people developed $\mathrm{TB}$ and 1.3 million died from the disease [1]. The number of TB deaths is unacceptably large given that most of these deaths are preventable. With the recent resurgence related to the human immunodeficiency virus (HIV), TB prevention and management has become further challenging [2-4]. TB is preventable as well as curable and its transmission could be prevented by prompt identification and treatment of the infected person. However; ensuring treatment completion is crucial for the prevention of relapse and secondary drug resistance.

\footnotetext{
* Correspondence: zulfiqar.bhutta@aku.edu

${ }^{2}$ Center of Excellence in Women \& Child Health, The Aga Khan University, Karachi, Pakistan

${ }^{3}$ Center for Global Child Health Hospital for Sick Children, Toronto, Canada Full list of author information is available at the end of the article
}

The World Health Organization (WHO) recommends Stop TB Strategy based on the Directly Observed Therapy, Short-course (DOTS) to control TB. The strategy aims to ensure that patients take a standard short-course of chemotherapy under guided supervision to cure the disease as well as to prevent transmission. Patients are assisted through their treatment regimen and encouraged to treatment completion in order to prevent resistance to the available anti-TB drugs. DOTS has been delivered by health workers, community volunteers, lay health workers and even family members [5]. For further details on TB burden, epidemiology and intervention coverage, refer to previous paper in this series [6].

Considering the recent shift in epidemiological presence of $\mathrm{TB}$, there is a legitimate call for integration of therapeutic services especially with HIV [7]. Since both diseases require long term treatment regimens, community based support may play a defining role towards prevention and control of these syndemic diseases of poverty. Moreover, integration of services in low-income countries may prove beneficial in terms of cost-effectiveness and decrease demand on health service infrastructure. However, there is a 
need to gauge whether these strategies lead to effective treatment outcomes. This paper aims to evaluate the effectiveness of community based interventions (CBI) for the prevention and treatment of TB.

\section{Methods}

We systematically reviewed literature published by September 2013 to identify studies evaluating CBI for TB as outlined in our conceptual framework [8]. Our priority was to select existing randomized controlled trials (RCT), quasiexperimental and before/after studies in which the intervention was delivered within community settings and the reported outcomes were relevant. A comprehensive search strategy was developed using appropriate key words, Medical Subject Headings (MeSH) and free text terms. The search was conducted in PubMed, Cochrane libraries, EMBASE and WHO Regional Databases; additional studies were identified by hand searching references from included studies. Studies were excluded if the intervention was purely facility-based or had a facility-based component. Studies that met the inclusion criteria were selected and double data abstracted on a standardized abstraction sheet. Quality assessment of the included RCT was done using the Cochrane risk of bias assessment tool [9]. We conducted meta-analysis for individual studies using the software Review Manager 5.1. Pooled statistics were reported as the relative risk (RR) for categorical variables and standard mean difference (SMD) for continuous variables between the experimental and control groups with 95\% confidence intervals (CI). Subgroup analysis was conducted for therapeutic and preventive (screening) CBI, integrated and non-integrated CBI and by type of studies. The detailed methodology is described in previous paper [8].

\section{Review}

A total of 7,772 titles were identified from all databases and 107 full texts were screened. After screening, forty one [10-50] studies met the inclusion criteria; 34 RCT and 7 before/after studies (Figure 1). From the included RCT,
18 were adequately randomized while five studies reported adequate sequence generation (Table 1). Due to the nature of the intervention, blinding of the participants and assessors was not possible. Studies provided insufficient information on selective reporting which limited us from making any judgment. Ten of the included studies focused on $\mathrm{TB}$ prevention and case detection while 31 studies were on treatment of patients with active TB. Interventions involved community based delivery of DOTS; community mobilization and support; education and training; and monetary incentives for treatment adherence. Most of the CBI utilized community health workers (CHW) or family members as part of the delivery strategy. Table 2 describes the characteristics of included studies.

\section{Quantitative synthesis}

Overall, CBI for TB prevention and case detection showed significant increase in TB detection rates (RR: 3.1, 95\% CI: $2.92,3.28$ ) (Figure 2) while there was a non-significant impact on TB incidence, although this evidence is from a single study. Subgroup analysis showed consistent results for various study designs and whether the interventions were delivered in an integrated or a non-integrated manner. CBI for treating patients with active TB showed an overall improvement in treatment success rates (RR: 1.09, 95\% CI: 1.07, 1.11) (Figure 3) and evidence from a single study suggests significant reduction in relapse rate (RR 0.26 , 95\% CI: $0.18,0.39$ ). The results were consistent for various study design and delivery mechanism. The results are summarized in Table 3.

\section{Qualitative synthesis}

Included studies suggest that CBI for TB have the potential to improve access to diagnostic and treatment services for poor rural communities and vulnerable population including women and children. Community based TB treatment delivery through CHW not only improved access and service utilization but also contributed to capacity building and improving routine TB recording and reporting

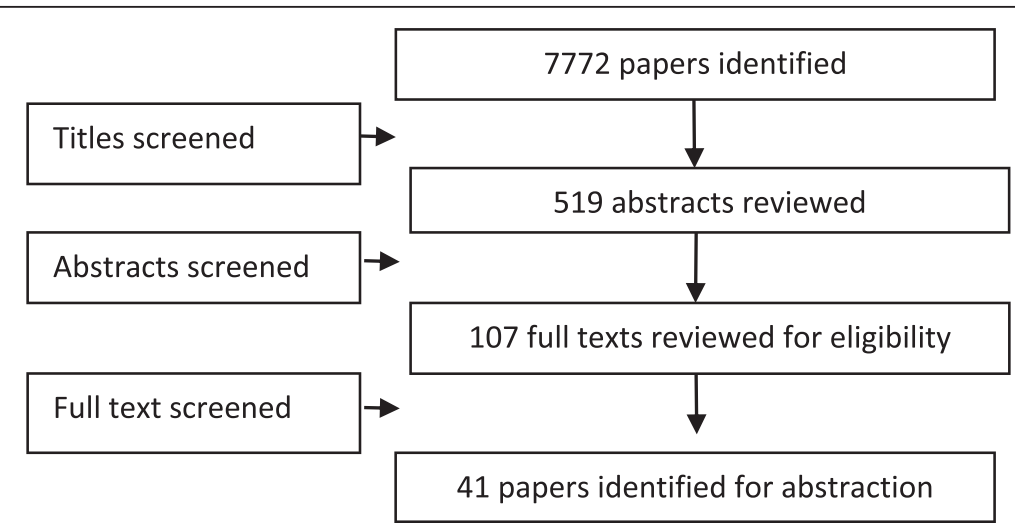

Figure 1 Search flow diagram. 
Table 1 Quality assessment of the included RCTs

\begin{tabular}{|c|c|c|c|c|c|c|}
\hline Article & Randomization & $\begin{array}{c}\text { Sequence } \\
\text { generation }\end{array}$ & $\begin{array}{c}\text { Allocation } \\
\text { concealment }\end{array}$ & $\begin{array}{l}\text { Blinding of } \\
\text { participants }\end{array}$ & $\begin{array}{l}\text { Blinding of } \\
\text { assessors }\end{array}$ & $\begin{array}{l}\text { Selective } \\
\text { reporting }\end{array}$ \\
\hline Atkins 2011 [11] & No & No & No & Not clear & Not clear & Not clear \\
\hline Barker 2002 [12] & No & No & Not clear & Not clear & Not clear & No \\
\hline Clarke 2005 [15] & Yes & No & Yes & No & No & No \\
\hline Colvin 2003 [16] & No & No & No & Not clear & Not clear & Yes \\
\hline Corbett 2010 [17] & Yes & Not clear & Yes & No & Yes & No \\
\hline Dudley 2003 [19] & No & No & No & Not clear & Not clear & No \\
\hline Fairall 2005 [20] & Yes & Yes & Not clear & No & Yes & No \\
\hline Ferreira 2011 [21] & No & No & No & Not clear & Not clear & No \\
\hline Filho 2009 [14] & No & No & No & Not clear & Not clear & Yes \\
\hline Kironde 2002 [23] & No & No & No & Not clear & Not clear & No \\
\hline Vieira 2011 [31] & No & No & No & Not clear & Not clear & Not clear \\
\hline White 2002 [33] & Yes & Yes & Yes & Not clear & Not clear & No \\
\hline Zwarenstein 2000 [35] & Yes & Yes & Yes & No & No & No \\
\hline Vassal 2002 [30] & Not clear & Not clear & Not clear & Not clear & Not clear & Not clear \\
\hline Prado 2011 [27] & No & No & No & No & No & No \\
\hline Mafigiri 2012 [24] & No & No & No & No & Not clear & No \\
\hline Niazi 2003 [26] & No & No & No & No & Not clear & No \\
\hline Uwimana 2012 [28] & Yes & Not clear & Not clear & Not clear & Not clear & No \\
\hline Yassin 2013 [34] & No & No & No & Not clear & Not clear & No \\
\hline CDI group & Yes & Not clear & Not clear & Not clear & Not clear & Not clear \\
\hline Miti 2003 [25] & No & No & No & Not clear & Not clear & No \\
\hline Zwarenstein 1998 [50] & Yes & Not clear & Not clear & No & No & No \\
\hline Chaisson 2001 [37] & Yes & Yes & Yes & Not clear & Not clear & No \\
\hline Heal 1998 [39] & No & No & No & No & No & No \\
\hline Kamolratanakul 1999 [40] & Yes & Yes & Yes & Yes & No & No \\
\hline Khan 2002 [41] & Yes & Not clear & Not clear & Not clear & Not clear & No \\
\hline Lwilla 2003 [42] & Yes & Not clear & Not clear & No & No & Yes \\
\hline Maclntyre 2003 [43] & No & No & No & No & Yes & Yes \\
\hline Malotte 2001 [44] & Yes & No & Yes & Not clear & Not clear & No \\
\hline Newell 2006 [45] & Yes & No & Yes & No & Yes & No \\
\hline Ollé-Goig 2001 [46] & No & No & No & No & No & No \\
\hline Walley 2001 [47] & Yes & Yes & Yes & No & Yes & No \\
\hline Wandwalo 2004 [48] & Yes & No & No & No & No & No \\
\hline Wright 2004 [49] & Yes & No & Yes & Not clear & Not clear & No \\
\hline
\end{tabular}

systems through regular supportive supervision [34]. Better outcomes were reported when DOTS was provided together with $\mathrm{CHW}$ program as it enabled treatment continuation; thus achieving higher treatment success rates [21]. Studies also support the feasibility of integrating cadres of CHW through establishing supportive structures and supervision [28,29]. Besides treatment, community based untargeted periodic active case finding for symptomatic smear-positive TB also made a substantial contribution to diagnosis and control of infectious TB [17]. This is a significant finding as the slow rate at which patients with tuberculosis report to health facilities is a major limitation in global efforts to control TB. However, especial emphasis needs to be given for training, close supervision and support for $\mathrm{CHW}$ to achieve job satisfaction and sustainability [34].

Despite considerable advocacy for increased collaboration and integration of TB and HIV care, few models of integration have been implemented, evaluated and reported $[11,17,20,28,29]$. However, existing evidence favors 


\begin{tabular}{|c|c|c|c|c|c|}
\hline Study & $\begin{array}{l}\text { Study } \\
\text { design }\end{array}$ & Country & Intervention & Target Population & $\begin{array}{l}\text { Integrated/ } \\
\text { Non-Integrated }\end{array}$ \\
\hline $\begin{array}{l}\text { Gandhi } 2008 \\
\text { [38] }\end{array}$ & $\begin{array}{l}\text { Before/ } \\
\text { after }\end{array}$ & South Africa & DOTS therapy integrated with anti-retroviral therapy on a community level & TB and HIV co-infected adults & Integrated \\
\hline $\begin{array}{l}\text { Chaisson } 2001 \\
{[37]}\end{array}$ & $\mathrm{RCT}$ & USA & Preventive Isoniazid therapy for injection drug users & Injection drug users & Non-integrated \\
\hline $\begin{array}{l}\text { Zwarenstein } \\
1998[50]\end{array}$ & RCT & South Africa & Self-supervised treatment compared against therapy observed in clinic & Adult pulmonary TB patients & Non-integrated \\
\hline Heal 1998 [39] & $\begin{array}{l}\text { Quasi- } \\
\text { trial }\end{array}$ & Canada & $\begin{array}{l}\text { Self-administered preventive therapy compared against preventive therapy observed in a } \\
\text { clinic }\end{array}$ & $\begin{array}{l}\text { All aboriginals in British Columbia } \\
\text { undergoing preventive therapy for TB }\end{array}$ & Non-integrated \\
\hline $\begin{array}{l}\text { Kamolratanakul } \\
1999[40]\end{array}$ & RCT & Thailand & DOTS therapy compared against self-supervised therapy & $\begin{array}{l}\text { All smear positive pulmonary TB } \\
\text { patients }\end{array}$ & Non-integrated \\
\hline Khan 2002 [41] & RCT & Pakistan & DOTS therapy compared against self-supervised therapy & Adults with TB & Non-integrated \\
\hline Lwilla 2003 [42] & RCT & Tanzania & Community-based DOTS compared against institution-based DOTS & $\begin{array}{l}\text { All patients diagnosed with TB at } \\
\text { selected health centres }\end{array}$ & Non-integrated \\
\hline $\begin{array}{l}\text { Maclntyre } 2003 \\
{[43]}\end{array}$ & $\begin{array}{l}\text { Quasi- } \\
\text { trial }\end{array}$ & Australia & Family member supervised DOTS compared against non-observed therapy & $\begin{array}{l}\text { All patients diagnosed with TB at } \\
\text { selected health centres }\end{array}$ & Non-integrated \\
\hline $\begin{array}{l}\text { Malotte } 2001 \\
{[44]}\end{array}$ & $\mathrm{RCT}$ & USA & INH therapy of latent TB infections given either by outreach workers or at a facility & $\begin{array}{l}\text { People with active or recent history of } \\
\text { drug use }\end{array}$ & Non-integrated \\
\hline $\begin{array}{l}\text { Newell } 2006 \\
{[45]}\end{array}$ & RCT & Nepal & Comparison between community-members DOTS and family member DOTS & $\begin{array}{l}\text { All new smear positive cases of } \\
\text { pulmonary TB }\end{array}$ & Non-integrated \\
\hline $\begin{array}{l}\text { Ollé-Goig } 2001 \\
\text { [46] }\end{array}$ & $\begin{array}{l}\text { Quasi- } \\
\text { trial }\end{array}$ & Haiti & DOTS compared with non-observed therapy & Adult TB patients & Non-integrated \\
\hline $\begin{array}{l}\text { Walley } 2001 \\
{[47]}\end{array}$ & RCT & Pakistan & $\begin{array}{l}\text { DOTS by family member compared with DOTS by healthcare worker and non-observed } \\
\text { therapy }\end{array}$ & Adult TB patients & Non-integrated \\
\hline $\begin{array}{l}\text { Wandwalo } \\
2004[48]\end{array}$ & RCT & Tanzania & Community-based DOTS compared against healthcare worker DOTS & TB patients of all ages & Non-integrated \\
\hline $\begin{array}{l}\text { Wright } 2004 \\
{[49]}\end{array}$ & RCT & Swaziland & Community health worker DOTS compared with family member DOTS & TB patients of all ages & Non-integrated \\
\hline Atkins 2011 [11] & $\begin{array}{l}\text { Quasi- } \\
\text { trial }\end{array}$ & South Africa & Enhanced tuberculosis treatment adherence & Adult TB patients & Integrated \\
\hline $\begin{array}{l}\text { Barker } 2002 \\
{[12]}\end{array}$ & $\begin{array}{l}\text { Quasi- } \\
\text { trial }\end{array}$ & South Africa & Community-based DOTS compared against healthcare worker DOTS & TB patients of all ages & Non-integrated \\
\hline Clarke 2005 [15] & RCT & South Africa & Comparison between conventional TB treatment and lay health worker DOTS & Adult TB patients & Non-integrated \\
\hline $\begin{array}{l}\text { Colvin } 2003 \\
{[16]}\end{array}$ & $\begin{array}{l}\text { Quasi- } \\
\text { trial }\end{array}$ & South Africa & Traditional healers mobilized as DOTS supervisors & TB patients of all ages & Non-integrated \\
\hline $\begin{array}{l}\text { Corbett } 2010 \\
{[17]}\end{array}$ & RCT & Zimbabwe & $\begin{array}{l}\text { Door-to-door and mobile van announcements compared as strategies to increase TB } \\
\text { detection }\end{array}$ & All people in a specific community & Integrated \\
\hline Diez 1996 [18] & $\begin{array}{l}\text { Before/ } \\
\text { after }\end{array}$ & Spain & Social support for deserving TB patients & Adult TB patients & Non-integrated \\
\hline
\end{tabular}


Table 2 Characteristics of the included studies (Continued)

\begin{tabular}{|c|c|c|c|c|c|}
\hline $\begin{array}{l}\text { Dudley } 2003 \\
\text { [19] }\end{array}$ & $\begin{array}{l}\text { Quasi- } \\
\text { trial }\end{array}$ & South Africa & DOTS compared with non-observed therapy & Adult TB patients & Non-integrated \\
\hline Fairall 2005 [20] & $\mathrm{RCT}$ & South Africa & Educational outreach to nurses to increase TB case detection & Patients attending specific clinics & Integrated \\
\hline $\begin{array}{l}\text { Ferreira } 2011 \\
{[21]}\end{array}$ & $\begin{array}{l}\text { Quasi- } \\
\text { trial }\end{array}$ & Brazil & DOTS compared with non-observed therapy & TB patients of all ages & Non-integrated \\
\hline Filho 2009 [14] & $\begin{array}{l}\text { Quasi- } \\
\text { trial }\end{array}$ & Brazil & Food baskets offered to patient to assess effect on treatment outcomes & Adult TB patients & Integrated \\
\hline $\begin{array}{l}\text { Kamineni } 2011 \\
{[22]}\end{array}$ & $\begin{array}{l}\text { Before/ } \\
\text { after }\end{array}$ & India & $\begin{array}{l}\text { Increasing case detection and treatment adherence, decreasing stigma and discrimination, } \\
\text { empowering affected people, and mobilising political commitment and resources }\end{array}$ & TB patients of all ages & Non-integrated \\
\hline $\begin{array}{l}\text { Kirondea } 2002 \\
{[23]}\end{array}$ & $\begin{array}{l}\text { Quasi- } \\
\text { trial }\end{array}$ & South Africa & Assessing the feasibility of using lay volunteers as DOTS supervisors & Adult TB patients & Non-integrated \\
\hline Vieira 2011 [31] & $\begin{array}{l}\text { Quasi- } \\
\text { trial }\end{array}$ & Brazil & DOTS compared with non-observed therapy & Adult TB patients & Non-integrated \\
\hline Weis 1994 [32] & $\begin{array}{l}\text { Before/ } \\
\text { after }\end{array}$ & USA & DOTS compared with non-observed therapy & TB patients of all ages & Non-integrated \\
\hline White 2002 [33] & $\mathrm{RCT}$ & USA & Incentivized treatment compared with no incentive & Susceptible population in a county jail & Non-integrated \\
\hline $\begin{array}{l}\text { Zwarenstein } \\
2000[35]\end{array}$ & RCT & South Africa & DOTS compared with non-observed therapy & TB patients of all ages & Non-integrated \\
\hline Prado $2011[27]$ & $\begin{array}{l}\text { Quasi- } \\
\text { trial }\end{array}$ & Brazil & Community health worker DOTS compared with family member DOTS & Adult TB patients & Non-integrated \\
\hline $\begin{array}{l}\text { Vassall } 2002 \\
{[30]}\end{array}$ & $\begin{array}{l}\text { Quasi- } \\
\text { trial }\end{array}$ & Syria and Egypt & Community-based DOTS compared against institution-based DOTS & TB patients of all ages & Integrated \\
\hline $\begin{array}{l}\text { CDI study } \\
\text { group }\end{array}$ & $\mathrm{RCT}$ & $\begin{array}{l}\text { Nigeria, Uganda } \\
\text { and Cameroon }\end{array}$ & $\begin{array}{l}\text { Integration of community interventions to counter multiple diseases through a single } \\
\text { framework }\end{array}$ & TB patients of all ages & Integrated \\
\hline Miti 2003 [25] & $\begin{array}{l}\text { Quasi- } \\
\text { trial }\end{array}$ & Zambia & Integration of HIV and TB services & Adult TB patients & Integrated \\
\hline $\begin{array}{l}\text { Amo-Adjei } \\
2013[10]\end{array}$ & $\begin{array}{l}\text { Before/ } \\
\text { after }\end{array}$ & Ghana & $\begin{array}{l}\text { Improvements in diagnosis, community TB care and stigma reduction among community and } \\
\text { health workers towards TB patients }\end{array}$ & Adult TB patients & Integrated \\
\hline Brust 2012 [13] & $\begin{array}{l}\text { Before/ } \\
\text { after }\end{array}$ & South Africa & Integration of HIV and TB services & Adult TB patients & Integrated \\
\hline $\begin{array}{l}\text { Mafigiri } 2012 \\
{[24]}\end{array}$ & $\begin{array}{l}\text { Quasi- } \\
\text { trial }\end{array}$ & Uganda & Community-based DOTS compared against institution-based DOTS & TB patients of all ages & Non-integrated \\
\hline Niazi 2003 [26] & $\begin{array}{l}\text { Quasi- } \\
\text { trial }\end{array}$ & Iraq & Community-based DOTS compared against institution-based DOTS & Adult TB patients & Non-integrated \\
\hline $\begin{array}{l}\text { Uwimana } 2012 \\
{[28]}\end{array}$ & $\mathrm{RCT}$ & South Africa & Training community care workers (CCWs) to provide integrated care & $\begin{array}{l}\text { All members of localities where the } \\
\text { CCWs were based }\end{array}$ & Integrated \\
\hline $\begin{array}{l}\text { Uwimana } 2013 \\
\text { [29] }\end{array}$ & $\begin{array}{l}\text { Before/ } \\
\text { after }\end{array}$ & South Africa & Training community care workers (CCWs) to provide integrated care & $\begin{array}{l}\text { All members of localities where the } \\
\text { CCWs were based }\end{array}$ & Integrated \\
\hline $\begin{array}{l}\text { Yassin } 2013 \\
{[34]}\end{array}$ & $\begin{array}{l}\text { Quasi- } \\
\text { trial }\end{array}$ & Ethiopia & $\begin{array}{l}\text { Training, engaging stakeholders and communities and active case-finding by female Health } \\
\text { Extension Workers (HEWs) at village level }\end{array}$ & $\begin{array}{l}\text { All members of localities where the } \\
\text { HEWs were based }\end{array}$ & Non-integrated \\
\hline
\end{tabular}




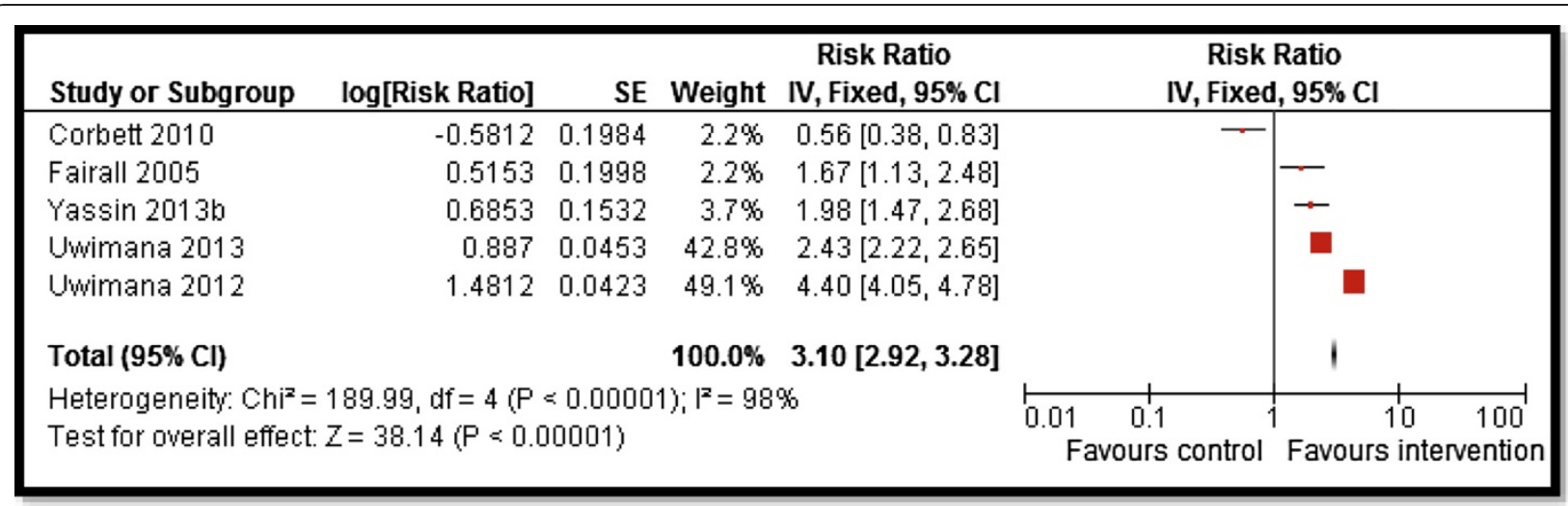

Figure 2 Forest plot for the impact of $\mathrm{CBI}$ on TB case detection.

integrating TB and HIV care for improving active case finding and early diagnosis of $\mathrm{TB}$, which in turn, reduces the risk of TB transmission [28]. TB/HIV co-infected patients receiving concurrent antiretroviral and TB therapy achieved high levels of adherence and excellent TB and
HIV outcomes [28]. Integrated provision of TB, HIV and prevention of mother to child transmission (PMTCT) services at community level through $\mathrm{CHW}$ is feasible, acceptable and successful $[28,29]$. Training CHW to provide a comprehensive package of TB HIVPMTCT prevention,

\begin{tabular}{|c|c|c|c|c|c|c|}
\hline \multirow{2}{*}{$\begin{array}{l}\text { Study or Subgroup } \\
\text { Amo-Adjei } 2013\end{array}$} & \multirow{2}{*}{$\begin{array}{r}\text { log[Risk Ratio] } \\
0.6817\end{array}$} & \multirow{2}{*}{$\begin{array}{r}\text { SE } \\
0.1193\end{array}$} & \multirow{2}{*}{$\frac{\text { Weight }}{0.5 \%}$} & \multirow{2}{*}{$\begin{array}{c}\begin{array}{c}\text { Risk Ratio } \\
\text { IV, Fixed, } 95 \% \mathrm{Cl}\end{array} \\
1.98[1.56,2.50]\end{array}$} & \multicolumn{2}{|c|}{$\begin{array}{c}\text { Risk Ratio } \\
\text { IV, Fixed, } 95 \% \mathrm{Cl}\end{array}$} \\
\hline & & & & & & $\longrightarrow$ \\
\hline Atkins 2011 & 0.0783 & 0.0291 & $7.7 \%$ & $1.08[1.02,1.14]$ & & $\rightarrow$ \\
\hline Barker 2002 & 0.0366 & 0.0327 & $6.1 \%$ & $1.04[0.97,1.11]$ & & \\
\hline Brust 2012 & 0.5596 & 0.1254 & $0.4 \%$ & $1.75[1.37,2.24]$ & & 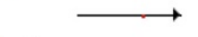 \\
\hline CDI study & 0.0183 & 0.061 & $1.7 \%$ & $1.02[0.90,1.15]$ & & \\
\hline Chaisson 2001 & 0.0101 & 0.0722 & $1.2 \%$ & $1.01[0.88,1.16]$ & & \\
\hline Clarke 2005 & 0.0773 & 0.0822 & $1.0 \%$ & $1.08[0.92,1.27]$ & & \\
\hline Colvin 2003 & 0.1498 & 0.0665 & $1.5 \%$ & $1.16[1.02,1.32]$ & & \\
\hline Dudley 2003 & 0.029 & 0.0414 & $3.8 \%$ & $1.03[0.95,1.12]$ & & \\
\hline Ferreira 2011 & 0.209 & 0.019 & $18.0 \%$ & $1.23[1.19,1.28]$ & & $\rightarrow$ \\
\hline Filho 2011 & 0.1977 & 0.0996 & $0.7 \%$ & $1.22[1.00,1.48]$ & & \\
\hline Gandhi 2008 & 0.3369 & 0.0909 & $0.8 \%$ & $1.40[1.17,1.67]$ & & \\
\hline Heal 1998 & -0.4836 & 0.093 & $0.7 \%$ & $0.62[0.51,0.74]$ & & \\
\hline Kamineni 2011 & 0.0085 & 0.021 & $14.7 \%$ & $1.01[0.97,1.05]$ & & - \\
\hline Kamolratanakul 1999 & 0.1016 & 0.0354 & $5.2 \%$ & $1.11[1.03,1.19]$ & & $\rightarrow$ \\
\hline Khan 2002 & 0.0776 & 0.1051 & $0.6 \%$ & $1.08[0.88,1.33]$ & & \\
\hline Kironde 2002 & 0.0428 & 0.0541 & $2.2 \%$ & $1.04[0.94,1.16]$ & & \\
\hline Lwilla 2003 & 0.0739 & 0.0864 & $0.9 \%$ & $1.08[0.91,1.28]$ & & \\
\hline Macintyre 2003 & -0.2089 & 0.0999 & $0.6 \%$ & $0.81[0.67,0.99]$ & & \\
\hline Mafigiri 2012 & -0.2437 & 0.1088 & $0.5 \%$ & $0.78[0.63,0.97]$ & & \\
\hline Malotte 2001 & -0.1273 & 0.1702 & $0.2 \%$ & $0.88[0.63,1.23]$ & & \\
\hline Miti 2003 & 0.2217 & 0.1404 & $0.3 \%$ & $1.25[0.95,1.64]$ & & \\
\hline Newell 2006 & 0.0507 & 0.0259 & $9.7 \%$ & $1.05[1.00,1.11]$ & & - \\
\hline Niazi 2003 & 0.1991 & 0.0871 & $0.9 \%$ & $1.22[1.03,1.45]$ & & \\
\hline Olle Goig 2001 & 0.4042 & 0.0784 & $1.1 \%$ & $1.50[1.28,1.75]$ & & \\
\hline Prado 2011 & 0.1603 & 0.0535 & $2.3 \%$ & $1.17[1.06,1.30]$ & & \\
\hline Vassal 2002a & 0.4787 & 0.0917 & $0.8 \%$ & $1.61[1.35,1.93]$ & & \\
\hline Vassal $2002 \mathrm{~b}$ & 0.1335 & 0.0988 & $0.7 \%$ & $1.14[0.94,1.39]$ & & \\
\hline Vieira 2011 & 0.0674 & 0.05 & $2.6 \%$ & $1.07[0.97,1.18]$ & & \\
\hline Walley 2001 & -0.0376 & 0.0837 & $0.9 \%$ & $0.96[0.82,1.13]$ & & \\
\hline Wandwalo 2004 & 0.0253 & 0.0362 & $4.9 \%$ & $1.03[0.96,1.10]$ & & \\
\hline White 2002 & 0.7092 & 0.338 & $0.1 \%$ & $2.03[1.05,3.94]$ & & 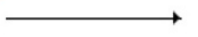 \\
\hline Wright 2004 & -0.0261 & 0.0383 & $4.4 \%$ & $0.97[0.90,1.05]$ & & \\
\hline Yassin 2013a & 0.1888 & 0.0612 & $1.7 \%$ & $1.21[1.07,1.36]$ & & \\
\hline Zwarenstein 1998 & 0.1044 & 0.1184 & $0.5 \%$ & $1.11[0.88,1.40]$ & & \\
\hline Zwarenstein 2000 & 0.2638 & 0.1398 & $0.3 \%$ & $1.30[0.99,1.71]$ & & \\
\hline Total $(95 \% \mathrm{Cl})$ & & & $100.0 \%$ & $1.09[1.07,1.11]$ & & 1 \\
\hline $\begin{array}{l}\text { Heterogeneity: } \mathrm{Chi}^{2}=2 \\
\text { Test for overall effect } Z\end{array}$ & $91, \mathrm{df}=35(\mathrm{P}=$ & 0.00001 ) & $1^{2}=85 \%$ & & 0.7 & 1.5 \\
\hline
\end{tabular}

Figure 3 Forest plot for the impact of $\mathrm{CBI}$ on treatment success rate. 
Table 3 Results for overall and sub-group analysis according to type of study, intervention and treatment

\begin{tabular}{|c|c|c|c|c|c|c|}
\hline \multirow[t]{2}{*}{ Outcomes } & \multicolumn{4}{|c|}{ Estimates $(95 \% \mathrm{Cl})$} & \multirow[b]{2}{*}{ Integrated } & \multirow[b]{2}{*}{ Non-integrated } \\
\hline & $\begin{array}{l}\text { Prevention/ } \\
\text { Screening }\end{array}$ & $\begin{array}{l}\text { Therapeutic } \\
\text { management }\end{array}$ & RCT/Quasi & Pre-post studies & & \\
\hline \multicolumn{7}{|c|}{ CBI for TB case detection } \\
\hline \multirow[t]{2}{*}{ TB detection } & $3.10[2.92,3.28]$ & - & $3.71[3.44,4.01]$ & $2.43[2.22,2.65]$ & $3.15[2.97,3.34]$ & $1.98[1.47,2.68]$ \\
\hline & 5 datasets, 5 studies & & $\begin{array}{l}4 \text { datasets, } 4 \\
\text { studies }\end{array}$ & 1 dataset, 1 study & $\begin{array}{l}4 \text { datasets, } 4 \\
\text { studies }\end{array}$ & 1 dataset, 1 study \\
\hline \multirow[t]{2}{*}{ TB incidence } & $0.63[0.36,1.09]$ & - & - & $0.63[0.36,1.09]$ & - & $0.63[0.36,1.09]$ \\
\hline & 1 dataset, 1 study & & & 1 dataset, 1 study & & 1 dataset, 1 study \\
\hline \multicolumn{7}{|c|}{ CBI for patients with Active TB } \\
\hline \multirow{2}{*}{$\begin{array}{l}\text { Treatment } \\
\text { success }\end{array}$} & - & $1.09[1.07,1.11]$ & $1.10[1.08,1.12]$ & $1.06[1.02,1.10]$ & $1.29[1.21,1.38]$ & $1.08[1.06,1.10]$ \\
\hline & & 36 datasets 35 studies & $\begin{array}{c}32 \text { datasets, } 31 \\
\text { studies }\end{array}$ & $\begin{array}{l}4 \text { datasets, } 4 \\
\text { studies }\end{array}$ & $\begin{array}{l}8 \text { datasets, } 7 \\
\text { studies }\end{array}$ & $\begin{array}{c}28 \text { datasets, } 28 \\
\text { studies }\end{array}$ \\
\hline \multirow[t]{2}{*}{ Relapse } & - & $0.26[0.18,0.39]$ & - & $0.26[0.18,0.39]$ & - & $0.26[0.18,0.39]$ \\
\hline & & 1 dataset, 1 study & & 1 dataset, 1 study & & 1 dataset, 1 study \\
\hline
\end{tabular}

Italics denote statistically significant estimates.

case finding and treatment support services can bridge the current gaps in service delivery through vertical TB, HIV and PMTCT programs. Evidence also suggests that DOTS strategy can be successfully implemented at primary health care clinics [31]. However such integrations should follow careful planning and caution with greater investments in developing and implementing infection control and laboratory infrastructure [28].

Key components reported for a successful community delivery strategy to prevent and treat TB included a preexisting TB DOTS infrastructure, patient treatment literacy training, and adherence support from trained $\mathrm{CHW}$ and family members [21]. Involvement of non-governmental organization (NGO) has also been reported as an essential component of TB programs [22]. Formation of community groups also have reported to contribute towards improved awareness and knowledge about TB and treatment adherence. Community groups help bridge gaps between health system and community through support and coordination. Multi-sectoral community mobilization events that engage community leaders is also one of the enabling tools for successful community based programs in TBHIVPMTCT care $[28,29]$. Engagement of successfully treated patients can also assist in reducing community stigma and discrimination [22]. Other strategies for organizing, coordinating and managing health care include continuous education and direct supervision of health providers; establishment of goals and regular monitoring of process and result indicators; and incentives for effective use of recommended guidelines [21].

One of the major reported barriers in the success of TB programs is non-adherence mainly due to the lack of support. The intensity of support for patients is reported to diminish in the continuation phase of treatment [11]. Lack of incentives, difficult treatment access, poor communication between health providers and patients, poor application of DOTS, lack of active search for missing patients, and limitations of supervision in treatment units are recognized barriers to treatment success [21]. In addition, the presence of multiple cadres of CHW providing TB and HIV services in silos has hindered the enhancement of collaborative TBHIV activities in community, as well as their supervision $[28,29]$. Inconsistency in the supply of commodities such as test kits need to be resolved to increase uptake of HIV testing and counseling $[28,29]$.

\section{Discussion}

Our review findings suggest that $\mathrm{CBI}$ are effective in $\mathrm{TB}$ detection and treatment but its role in preventing $\mathrm{TB}$ cases has not been comprehensively evaluated. Community based delivery of DOTS may be more feasible and effective for $\mathrm{TB}$ case detection and treatment as community workers are familiar with the layout of community and have community member's trust which healthcare officials would have to develop. Moreover, a community-based approach helps empower each community to deal with its own problems and also provides patient with a greater degree of autonomy and satisfaction with the treatment regime [51]. This involvement of respected, responsible and resourceful community and family members increases the trust that is required to initiate treatment and provides close supervision thereby maximizing adherence which is crucial in such a lengthy treatment regime. Limited coverage of public health services has continued to impede accelerated access to TB control services due to inadequate health service infrastructure, insufficient decentralization of services and inadequate human, material and financial resources. Hence, community delivery platforms offer improved access and equitable distribution of care. 
High incidence of TB and its significant financial burden makes it imperative to find a plausible strategy to cope with this disease. The fact that it effects lower socio-economic groups further compounds the problem. Gender inequality, social stigma, and poverty are also recognized as important barriers for successful TB prevention and control programs [52-55]. In light of the above situation, DOTS provides a successful and costeffective strategy to deal with the burden of $\mathrm{TB}$ $[27,30,41]$. CBI coupled with DOTS seems to be an effective approach as they have the potential to maximize the outreach and minimize the cost. Community based TB control also offers many lessons for the control of HIV epidemic. With the emergence of HIV and consequent $\mathrm{TB}$ resurgence, a comprehensive and equitable strategy is needed to stem the worsening double burden of these two infections in poor countries [56].

The WHO currently advocates home-based care and integrated management of dually infected TB/AIDS patients [57]. It recommends a 12 point package of collaborative TB/HIV activities based on creating a mechanism of collaboration between TB and HIV programs, reducing the burden of TB among people living with HIV and reducing the burden of HIV among TB patients. CHW delivering DOTS can be further trained to carry out this additional task and studies are needed to evaluate the feasibility, relative effectiveness and cost effectiveness of this approach [23,58]. However, such integration would involve $\mathrm{CHW}$ training and time; improved collaboration between community and facility; and strengthening referral services $[59,60]$.

\section{Conclusion}

Well-designed operational research is needed to pragmatically evaluate various models of community based delivery. There is a need to evaluate and address context specific barriers to community based implementation, especially for collaborative TBHIV activities in the community to avoid duplication of labor and resources. Future studies should focus on evaluating novel community delivery models for their success in larger and more diverse populations and impact TB prevention and active case detection.

\section{Additional file}

Additional file 1: Multilingual abstracts in the six official working languages of the United Nations.

\footnotetext{
Abbreviations

CBI: Community based interventions; CHW: Community health workers; Cl: Confidence interval; DOTS: Direct observed therapy; HIV: Human immunodeficiency virus; MDR-TB: Multi drug resistant tuberculosis; NGO: Non-governmental organization; PMTCT: Prevention of mother-to-child transmission; RCT: Randomized controlled trial; RR: Relative risk; SMD: Standard mean difference; TB: Tuberculosis; WHO: World Health organization
}

\section{Competing interest}

The authors declare that they have no financial or non-financial competing interests.

\section{Authors' contribution}

$Z A B$ was responsible for designing and coordinating the review. AA and IN were responsible for: data collection, screening the search results, screening retrieved papers against inclusion criteria, appraising quality of papers and abstracting data. RAS, JKD and ZSL were responsible for data interpretation and writing the review. ZAB critically reviewed and modified the manuscript. All authors read and approved the final manuscript.

\section{Acknowledgements}

The collection of scoping reviews in this special issue of Infectious Diseases of Poverty was commissioned by the UNICEF/UNDP/World Bank/WHO Special Programme for Research and Training in Tropical Diseases (TDR) in the context of a Contribution Agreement with the European Union for "Promoting research for improved community access to health interventions in Africa".

\section{Author details}

${ }^{1}$ Division of Women and Child Health, The Aga Khan University, 74800 Karachi, Pakistan. ${ }^{2}$ Center of Excellence in Women \& Child Health, The Aga Khan University, Karachi, Pakistan. ${ }^{3}$ Center for Global Child Health Hospital for Sick Children, Toronto, Canada.

Received: 5 January 2014 Accepted: 6 June 2014

Published: 1 August 2014

\section{References}

1. World Health Organization: Global Tuberculosis Report; 2013. Availabel at http:// apps.who.int/iris/bitstream/10665/91355/1/9789241564656_eng.pdf?ua=1.

2. WHO: Global Tuberculosis Control: Epidemiology, Strategy, Financing: WHO, 2009. Geneva, Switzerland: WHO; 2009.

3. Rose AM, Watson JM, Graham C, Nunn AJ, Drobniewski F, Ormerod LP, Darbyshire $\mathrm{JH}$, Leese J: Tuberculosis at the end of the 20th century in England and Wales: results of a national survey in 1998. Thorax 2001, 56(3):173-179.

4. Schluger NW, Burzynski J: Tuberculosis and HIV infection: epidemiology, immunology, and treatment. HIV Clin Trials 2001, 2(4):356-365.

5. WHO: Community contribution to TB care: practice and policy. In WHO. Geneva: World Health Organization; 2003.

6. Bhutta ZA, Sommerfeld J, Lassi ZS, Salam RA, Das JK: Global burden, distribution and interventions for the infectious diseases of poverty. Infect Dis Pov 2014, 3:21.

7. Wood R: The case for integrating tuberculosis and HIV treatment services in South Africa. J Infect Dis 2007, 196(Suppl 3):S497-S499.

8. Lassi ZS, Salam RA, Das JK, Bhutta ZA: Conceptual framework and assessment methodology for the systematic review on community based interventions for the prevention and control of IDoP. Infect Dis PoV 2014, 3:22

9. Higgins JPT, Green S: Cochrane Handbook for Systematic Reviews of Interventions Version 5.1.0. [updated March 2011]. The Cochrane Collaboration, 2011. Available from [www.cochrane-handbook.org]

10. Amo-Adjei J, Awusabo-Asare K: Reflections on tuberculosis diagnosis and treatment outcomes in Ghana. Arch Public Health 2013, 71(1):22.

11. Atkins S, Lewin S, Jordaan E, Thorson A: Lay health worker-supported tuberculosis treatment adherence in South Africa: an interrupted timeseries study. Int J Tuberc Lung Dis 2011, 15(1):84-89. i.

12. Barker RD, Millard FJ, Nthangeni ME: Unpaid community volunteerseffective providers of directly observed therapy (DOT) in rural South Africa. S Afr Med J 2002, 92(4):291-294.

13. Brust JC, Shah NS, Scott M, Chaiyachati K, Lygizos M, van der Merwe TL, Bamber S, Radebe Z, Loveday M, Moll AP, Margot B, Lalloo UG, Friedland $\mathrm{GH}$, Gandhi NR: Integrated, home-based treatment for MDR-TB and HIV in rural South Africa: an alternate model of care. Int J Tuberc Lung Dis 2012, 16(8):998-1004.

14. Cantalice Filho JP: Food baskets given to tuberculosis patients at a primary health care clinic in the city of Duque de Caxias, Brazil: effect on treatment outcomes. J Bras Pneumol 2009, 35(10):992-997. 
15. Clarke M, Dick J, Zwarenstein M, Lombard CJ, Diwan VK: Lay health worker intervention with choice of DOT superior to standard TB care for farm dwellers in South Africa: a cluster randomised control trial. Int J Tuberc Lung Dis 2005, 9(6):673-679.

16. Colvin M, Gumede L, Grimwade K, Maher D, Wilkinson D: Contribution of traditional healers to a rural tuberculosis control programme in Hlabisa, South Africa. Int J Tuberc Lung Dis 2003, 7(9 Suppl 1):S86-S91.

17. Corbett EL, Bandason T, Duong T, Dauya E, Makamure B, Churchyard GJ, Williams BG, Munyati SS, Butterworth AE, Mason PR, Mungofa S, Hayes RJ: Comparison of two active case-finding strategies for community-based diagnosis of symptomatic smear-positive tuberculosis and control of infectious tuberculosis in Harare, Zimbabwe (DETECTB): a clusterrandomised trial. Lancet 2010, 376(9748):1244-1253.

18. Diez E, Claveria J, Serra T, Cayla JA, Jansa JM, Pedro R, Villalbi JR: Evaluation of a social health intervention among homeless tuberculosis patients. Tuber Lung Dis 1996, 77(5):420-424

19. Dudley L, Azevedo V, Grant R, Schoeman JH, Dikweni L, Maher D: Evaluation of community contribution to tuberculosis control in Cape Town, South Africa. Int J Tuberc Lung Dis 2003, 7(9 Suppl 1):S48-S55.

20. Fairall LR, Zwarenstein M, Bateman ED, Bachmann M, Lombard C, Majara BP, Joubert G, English RG, Bheekie A, van Rensburg D, Mayers P, Peters AC, Chapman RD: Effect of educational outreach to nurses on tuberculosis case detection and primary care of respiratory illness: pragmatic cluster randomised controlled trial. BMJ 2005, 331(7519):750-754.

21. Ferreira V, Brito C, Portela M, Escosteguy C, Lima S: DOTS in primary care units in the city of Rio de Janeiro. Southeastern Brazil Rev Saude Publica 2011, 45(1):40-48.

22. Kamineni W, Turk T, Wilson N, Satyanarayana S, Chauhan LS: A rapid assessment and response approach to review and enhance advocacy, communication and social mobilisation for tuberculosis control in Odisha state. India BMC Public Health 2011, 11:463

23. Kironde S, Kahirimbanyi M: Community participation in primary health care (PHC) programmes: lessons from tuberculosis treatment delivery in South Africa. Afr Health Sci 2002, 2(1):16-23.

24. Mafigiri DK, McGrath JW, Whalen CC: Task shifting for tuberculosis control: a qualitative study of community-based directly observed therapy in urban Uganda. Glob Public Health 2012, 7(3):270-284.

25. Miti S, Mfungwe $V$, Reijer $P$, Maher D: Integration of tuberculosis treatment in a community-based home care programme for persons living with HIV/AIDS in Ndola. Zambia Int J Tuberc Lung Dis 2003, 7(9 Suppl 1):S92-S98.

26. Niazi AD, Al-Delaimi AM: Impact of community participation on treatment outcomes and compliance of DOTS patients in Iraq. East Mediterr Health J 2003, 9(4):709-717

27. Prado TN, Wada N, Guidoni LM, Golub JE, Dietze R, Maciel EL: Costeffectiveness of community health worker versus home-based guardians for directly observed treatment of tuberculosis in Vitoria, Espirito Santo State. Brazil Cad Saude Publica 2011, 27(5):944-952.

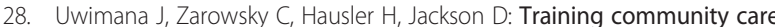
workers to provide comprehensive TB/HIV/PMTCT integrated care in KwaZulu-Natal: lessons learnt. Trop Med Int Health 2012, 17(4):488-496.

29. Uwimana J, Zarowsky C, Hausler H, Swanevelder S, Tabana H, Jackson D: Community-based intervention to enhance provision of integrated TB-HIV and PMTCT services in South Africa. Int J Tuberc Lung Dis 2013, 17(10 Suppl 1):48-55.

30. Vassall A, Bagdadi S, Bashour H, Zaher H, Maaren PV: Cost-effectiveness of different treatment strategies for tuberculosis in Egypt and Syria. Int $J$ Tuberc Lung Dis 2002, 6(12):1083-1090

31. Vieira AA, Ribeiro SA: Compliance with tuberculosis treatment after the implementation of the directly observed treatment, short-course strategy in the city of Carapicuiba. Brazil J Bras Pneumol 2011, 37(2):223-231.

32. Weis SE, Slocum PC, Blais FX, King B, Nunn M, Matney GB, Gomez E, Foresman $\mathrm{BH}$ : The effect of directly observed therapy on the rates of drug resistance and relapse in tuberculosis. N Engl J Med 1994, 330(17):1179-1184.

33. White MC, Tulsky JP, Goldenson J, Portillo CJ, Kawamura M, Menendez E: Randomized controlled trial of interventions to improve follow-up for latent tuberculosis infection after release from jail. Arch Intern Med 2002, 162(9):1044-1050.

34. Yassin MA, Datiko DG, Tulloch O, Markos P, Aschalew M, Shargie EB, Dangisso MH, Komatsu R, Sahu S, Blok L, Cuevas LE, Theobald S: Innovative community- based approaches doubled tuberculosis case notification and improve treatment outcome in Southern Ethiopia. PLoS One 2013, 8(5):e63174

35. Zwarenstein M, Schoeman JH, Vundule C, Lombard CJ, Tatley M: A randomised controlled trial of lay health workers as direct observers for treatment of tuberculosis. Int J Tuberc Lung Dis 2000, 4(6):550-554.

36. Group C: Community-directed interventions for priority health problems in Africa: results of a multicountry study. Bull World Health Organ 2010, 88(7):509-518

37. Chaisson RE, Barnes GL, Hackman J, Watkinson L, Kimbrough L, Metha S, Cavalcante S, Moore RD: A randomized, controlled trial of interventions to improve adherence to isoniazid therapy to prevent tuberculosis in injection drug users. Am J Med 2001, 110(8):610-615.

38. Gandhi NR, Moll AP, Lalloo U, Pawinski R, Zeller K, Moodley P, Meyer E, Friedland G: Successful integration of tuberculosis and HIV treatment in rural South Africa: the Sizonq'oba study. J Acquir Immune Defic Syndr 2009, 50(1):37-43

39. Heal G, Elwood RK, FitzGerald JM: Acceptance and safety of directly observed versus self-administered isoniazid preventive therapy in aboriginal peoples in British Columbia. Int J Tuberc Lung Dis 1998, 2(12):979-983.

40. Kamolratanakul P, Sawert H, Lertmaharit S, Kasetjaroen Y, Akksilp S, Tulaporn C, Punnachest K, Na-Songkhla S, Payanandana V: Randomized controlled trial of directly observed treatment (DOT) for patients with pulmonary tuberculosis in Thailand. Trans $R$ Soc Trop Med Hyg 1999, 93(5):552-557

41. Khan MA, Walley JD, Witter SN, Imran A, Safdar N: Costs and cost-effectiveness of different DOT strategies for the treatment of tuberculosis in Pakistan. Directly Observed Treatment. Health Policy Plan 2002, 17(2):178-186.

42. Lwilla F, Schellenberg D, Masanja H, Acosta C, Galindo C, Aponte J, Egwaga S, Njako B, Ascaso C, Tanner M, Alonso P: Evaluation of efficacy of community-based vs. institutional-based direct observed short-course treatment for the control of tuberculosis in Kilombero district, Tanzania. Trop Med Int Health 2003, 8(3):204-210.

43. Maclntyre CR, Goebel K, Brown GV, Skull S, Starr M, Fullinfaw RO: A randomised controlled clinical trial of the efficacy of family-based direct observation of anti-tuberculosis treatment in an urban, developedcountry setting. Int J Tuberc Lung Dis 2003, 7(9):848-854.

44. Malotte CK, Hollingshead JR, Larro M: Incentives vs outreach workers for latent tuberculosis treatment in drug users. Am J Prev Med 2001, 20(2):103-107.

45. Newell JN, Baral SC, Pande SB, Bam DS, Malla P: Family-member DOTS and community DOTS for tuberculosis control in Nepal: cluster-randomised controlled trial. Lancet 2006, 367(9514):903-909.

46. Olle-Goig JE, Alvarez J: Treatment of tuberculosis in a rural area of Haiti: directly observed and non-observed regimens. The experience of $\mathrm{H}$ pital Albert Schweitzer. Int J Tuberc Lung Dis 2001, 5(2):137-141.

47. Walley JD, Khan MA, Newell JN, Khan MH: Effectiveness of the direct observation component of DOTS for tuberculosis: a randomised controlled trial in Pakistan. Lancet 2001, 357(9257):664-669.

48. Wandwalo E, Kapalata N, Egwaga S, Morkve O: Effectiveness of community-based directly observed treatment for tuberculosis in an urban setting in Tanzania: a randomised controlled trial. Int J Tuberc Lung Dis 2004, 8(10):1248-1254.

49. Wright J, Walley J, Philip A, Pushpananthan S, Dlamini E, Newell J, Dlamini S: Direct observation of treatment for tuberculosis: a randomized controlled trial of community health workers versus family members. Trop Med Int Health 2004, 9(5):559-565.

50. Zwarenstein M, Schoeman JH, Vundule C, Lombard CJ, Tatley M: Randomised controlled trial of self-supervised and directly observed treatment of tuberculosis. Lancet 1998, 352(9137):1340-1343.

51. WHO: Community Involvement in Tuberculosis Care and Prevention. Towards Partnerships for Health; 2008. Available at http://whqlibdoc.who.int/ publications/2008/9789241596404_eng.pdf.

52. Atre SR, Kudale AM, Morankar SN, Rangan SG, Weiss MG: Cultural concepts of tuberculosis and gender among the general population without tuberculosis in rural Maharashtra. India Trop Med Int Health 2004 9(11):1228-1238.

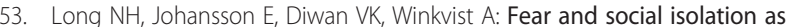
consequences of tuberculosis in VietNam: a gender analysis. Health Policy 2001, 58(1):69-81.

54. Weiss MG, Somma D, Karim F, Abouihia A, Auer C, Kemp J, Jawahar MS: Cultural epidemiology of TB with reference to gender in Bangladesh, India and Malawi. Int J Tuberc Lung Dis 2008, 12(7):837-847. 
55. Aryal S, Badhu A, Pandey S, Bhandari A, Khatiwoda P, Khatiwada P, Giri A: Stigma related to tuberculosis among patients attending DOTS clinics of Dharan municipality. Kathmandu Univ Med J (KUMJ) 2012, 10(37):48-52.

56. Farmer P, Laandre F, Mukherjee J, Gupta R, Tarter L, Kim JY: Communitybased treatment of advanced HIV disease: introducing DOT-HAART (directly observed therapy with highly active antiretroviral therapy). Bull World Health Organ 2001, 79(12):1145-1151.

57. World Health Organization: WHO Policy on Collaborative TB/HIV Activities: Guidelines for National Programmes and other Stakeholders. Geneva: World Health Organization; 2012.

58. Kironde S: Tuberculosis. In South African Health Review. Edited by Ntuli A, Crisp N, Clarke E, Baron P. Durban: Health Systems Trust; 2000:335-349.

59. Legido-Quigley H, Montgomery CM, Khan P, Atun R, Fakoya A, Getahun H, Grant AD: Integrating tuberculosis and HIV services in low and middleincome countries: a systematic review. Trop Med Int Health 2013, 18(2):199-211

60. Howard AA, El-Sadr WM: Integration of tuberculosis and HIV services in sub-Saharan Africa: lessons learned. Clin Infect Dis 2010, 50(Supplement 3):S238-S244.

doi:10.1186/2049-9957-3-27

Cite this article as: Arshad et al:: Community based interventions for the prevention and control of tuberculosis. Infectious Diseases of Poverty 2014 3:27.

\section{Submit your next manuscript to BioMed Central} and take full advantage of:

- Convenient online submission

- Thorough peer review

- No space constraints or color figure charges

- Immediate publication on acceptance

- Inclusion in PubMed, CAS, Scopus and Google Scholar

- Research which is freely available for redistribution 